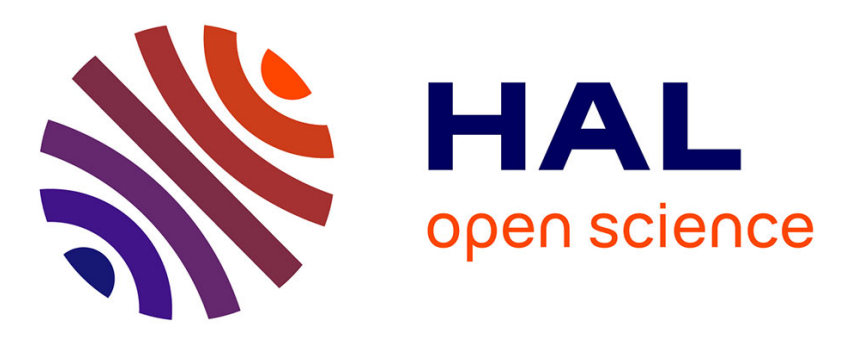

\title{
Vertically oriented nickel nanorod/carbon nanofiber core/shell structures synthesized by plasma-enhanced chemical vapor deposition
}

Zanbing He, Chang Seok Lee, Jean Luc Maurice, Didier Pribat, Paul Haghi-Ashtiani, Costel Sorin Cojocaru

\section{To cite this version:}

Zanbing He, Chang Seok Lee, Jean Luc Maurice, Didier Pribat, Paul Haghi-Ashtiani, et al.. Vertically oriented nickel nanorod/carbon nanofiber core/shell structures synthesized by plasma-enhanced chemical vapor deposition. Carbon, 2011, 49 (14), pp.4710-4718. 10.1016/j.carbon.2011.06.075 . hal-00793979

\section{HAL Id: hal-00793979 \\ https://hal.science/hal-00793979}

Submitted on 2 Mar 2013

HAL is a multi-disciplinary open access archive for the deposit and dissemination of scientific research documents, whether they are published or not. The documents may come from teaching and research institutions in France or abroad, or from public or private research centers.
L'archive ouverte pluridisciplinaire HAL, est destinée au dépôt et à la diffusion de documents scientifiques de niveau recherche, publiés ou non, émanant des établissements d'enseignement et de recherche français ou étrangers, des laboratoires publics ou privés. 


\title{
CARBON
}

\author{
vol 49 ( 2011) pages $4710-4718$ \\ DOI: http://dx.doi.org/10.1016/j.carbon.2011.06.075
}

\section{Vertically oriented nickel nanorod/carbon nanofiber core/shell structures synthesized by plasma-enhanced chemical vapor deposition}

\author{
Zhanbing He a,, , Chang Seok Lee a, Jean-Luc Maurice a, Didier Pribat a,b,*, Paul Haghi-Ashtiani c, Costel \\ Sorin Cojocaru a
}

a Laboratoire de Physique des Interfaces et Couches Minces (LPICM), UMR 7647, Ecole Polytechnique-CNRS, Route de Saclay,

91128 Palaiseau Cedex, France

๖ Department of Energy Science, Sungkyunkwan University, Suwon 440-746, Republic of Korea

c Laboratoire de Mecanique des Sols, Structures et Mate riaux (CNRS), UMR 8579, Ecole Centrale Paris, France

\begin{abstract}
A BSTRACT
Plasma-enhanced chemical vapor deposition, without a nickel-containing gaseous precur-sor, was used to synthesize continuous nickel (Ni) nanorods inside the hollow cavity of car- bon nanofibers (CNFs), thus forming vertically aligned Ni/CNF core/shell structures. Scanning and transmission electron microscopic images indicate that the elongated Ni nanorods originate from the catalyst particles at the tips of the CNFs and that their forma- tion is due to the effect of extrusion induced by the compressive force of the graphene lay- ers during growth. Different from previous work, each vertically-aligned core/shell structure reported is totally isolated from its neighbors. Continuous $\mathrm{Ni}$ nanorods are found to separate into smaller ones with increasing growth time, which was ascribed to (i) the limited amount of Ni available in the tip of the CNF, (ii) the polycrystalline nature of the Ni nanorods and (iii) the combined effects of the compressive stresses on the side of the Ni nanorods and of the tensile stress along their axis.
\end{abstract}

\section{Introduction}

Since the discovery of carbon nanotubes (CNTs), the inner tubular cavity of CNTs/carbon nanofibers (CNFs) has been witnessed to host nanowires, or nanoparticles that can be used for a wide variety of applications in biology, medicine, and nanodevices [1-15]. Till now, CNTs/CNFs with confined metal/alloy nanowires or nanoparticles have been produced by the arc-discharge method [4], detonation-assisted chemi- cal vapor deposition [5], and more often, by feeding catalytic precursors during CVD growth [3,6-9]. Although the majority of metal/CNT core/shell hybrids were randomly organized, vertically aligned metal/CNT core/shell nanostructures have been reported $[3,6,13,16]$. However, the alignment of the CNTs was caused by the crowding effect, in which each CNT is in close van der Waals contact with its neighbors during growth, which could bring technical troubles for CNT separation in the following steps. On the other hand, metal particles/nanorods encapsulated in CNTs are frequently broken into several pieces and spread along the tube length, which makes it challenging to synthesize continuous metal nanorods/nano- wires in vertically aligned CNTs/CNFs with their whole cavity filled. Nickel, as a magnetic material, has been explored for filling the cores of CNTs/CNFs, in order to realize functional- ities with some unique advantages, such as enhanced magnetic coercivity or anti-oxidation barrier for long-term stability. Recently, Nickel filling in the core of CNTs was re- ported by using Ni(salen) precursor, and a conventional pho- toresist during a CVD process [11], but the orientations of CNTs were not controlled. Moreover, Ni only filled-in discon- tinuous parts of CNTs, leaving some empty space in their hollow cavities [11]. Plasma-enhanced chemical vapor depo- sition (PECVD) is known to be a practical way to synthesize vertically-aligned CNTs/CNFs [16,17], and Ni has been used as catalyst for growing vertically aligned CNFs $[18,19]$. How- ever, no Ni filling was observed in the core of CNFs $[18,19]$. To the best of our knowledge, there is no report on the syn- thesis of continuous filling of $\mathrm{Ni}$ rods inside the whole cavity of CNTs/CNFs to date. Herein, we present a facile method to synthesize vertically aligned Ni/CNF core/shell structures, with continuous Ni nanorods filling the whole cavity of CNFs. More importantly, each metal/CNT is completely isolated from its neighbors, which could facilitate the disper- sion in subsequent processes or be useful for some applica- tions in nanomagnetism or spintronics. Moreover, in order to study the formation mechanism of Ni nanorods confined in- side CNFs, we have carried out a systematic study by ex-situ transmission electron microscopy (TEM) on samples grown by PECVD at 650 oC for three different growth times. Our re- sults not only show an efficient method to synthesize verti- cally aligned Ni/CNF core/shell nanostructures, but also give some insights for understanding the origin of residual cata- lysts in the core of CNTs, which are commonly found in CNTs grown by catalytic CVD. 


\section{Experimental details}

Vertically aligned CNFs arrays were grown in a home-made dc PECVD system, from 30-nm thick Ni catalyst layers evapo- rated on oxidized Si wafers. The PECVD system comprises three independent electrodes. A dc plasma is generated be- tween two mesh electrodes made of graphite and ions are ex- tracted from the plasma, down to the substrate, by negatively biasing the substrate holder (third electrode) with respect to the cathode. The growth temperature was set at 650 oC with different growth times: 10, 20, and 85 min. A mixture of water vapor $\left(\mathrm{H}_{2} \mathrm{O}\right)$ and isopropyl alcohol $\left(\mathrm{C}_{2} \mathrm{H}_{2} \mathrm{OH}\right)$ was used as the reactive gas source and the gas flows were precisely con- trolled to get a $\mathrm{C} / \mathrm{H}$ ratio of 0.2647 , in order to avoid the para- sitic deposition of amorphous carbon on the surface of the catalysts. The applied voltage between anode and cathode in the chamber was kept at a constant value of $500 \mathrm{~V}$, and with a current intensity of $0.2 \mathrm{~A}$. The extraction voltage and current were adjusted and stabilized at $54 \mathrm{~V}$ and $54 \mathrm{~mA}$, respectively. At the end of the growth process, the chamber was totally pumped out and cooled down. Cooling took around $30 \mathrm{~min}$ without any gas circulating in the growth chamber. An Hitachi S-4800 FE-SEM working at $25 \mathrm{kV}$ was employed to investigate the morphology of the asgrown samples. A Philips CM 30 transmission electron microscope equipped with a LaB 6 filament, operated at $300 \mathrm{kV}$, was used to study the microstructures of $\mathrm{Ni} / C N F s$ core/shell structures. A $0.5 \mathrm{Im}$ objective aperture was used for dark-field imaging.

\section{Results and discussion}

Fig. 1 shows scanning electron microscopy (SEM) images of vertically-aligned Ni/CNFs core/shell structures grown during different times. Core/shell structures are clearly revealed by dark (Ni) and light gray (CNFs) contrast from the high magni- fication images of Fig. $1 \mathrm{~d}$ and $\mathrm{f}$ in the right hand side column. Nickel particles in Fig. $1 \mathrm{a}$ and $\mathrm{b}$ are progressively elongated along the axis of CNFs to form Ni nanorods when the growth time is increased to $20 \mathrm{~min}$ (Fig. 1c and d). A majority of Ni nanorods are continuous, having a tadpole-shape with their big heads at the tips of CNFs and long tails encapsulated in the cavity of CNFs. When further increasing the growth time, e.g., to $85 \mathrm{~min}$ (Fig. 1e and f), the continuous Ni nanorods (Fig. 1c and d) were broken into several smaller individual ones, while the lateral contact with the innermost graphene layers of CNFs was maintained. TEM was used to investigate the core/shell nanostructures. Fig. 2 is a bright-field image of CNFs grown for 20 min. From the left hand side of Fig. 2a, it clearly appears that Ni nano- rods are filling the whole inner cavity of CNFs, without any space in the core of the thick CNFs. For example, a thick CNF with an external diameter of rv250 $\mathrm{nm}$ is filled with a long Ni nanorod exhibiting a length up to $1.15 \mathrm{~lm}$ and forming a core/shell structure, as marked in Fig. 2a, left hand side. How- ever, Ni nanorods are seldom found to be encapsulated in thinner CNFs with an outer diameter less than $200 \mathrm{~nm}$, e.g., no Ni nanorod is observed in the core of the thin CNF with a diameter of $50 \mathrm{~nm}$, as seen in the right hand side of Fig. 2a. The dark and bright contrast of the long $\mathrm{Ni}$ nanorods in Fig. $2 \mathrm{a}$ is not constant, implying that the long $\mathrm{Ni}$ nanorods should be polycrystalline, which will be verified by a careful TEM characterization in Fig. 3. Seventy-eight (78) individual CNFs were selected on ran- domly picked specimens for statistical analysis. 56 out of 78 CNFs were found to be filled with Ni nanorods, i.e., rv70\%. Moreover, rv91\% of those 56 Ni nanorods are continuous. It is worth noting that the filling of CNFs by nanorods is diame- ter dependent (Fig. 2b): the average outer diameter of filled CNFs is 0.28 Im, while that of empty CNFs is $0.15 \mathrm{~lm}$ (Fig. 2b). Moreover, $96 \%$ of the filled CNFs have an external diameter larger than $0.2 \mathrm{Im}$. As a comparison, $87 \%$ CNFs have an external diameter smaller than $0.2 \mathrm{~lm}$ among the CNFs unfilled with Ni. However, the inner diameter of the large CNFs is much smaller than outer diameter (Fig. 2b). Therefore, when the catalyst particle exhibits a large volume, it can oc- cupy more space in the core of the CNF, which explains why Ni rods appear in those CNFs with large outer diameters. The inner diameter of the small CNFs is smaller than that of the large ones, but the catalyst particles are too small to be filled in the core of small CNFs. Normally, Ni nanorods are somewhat shorter than their hosting CNF, due to some thick graphite-like layers occupying the base of the latter, as previously observed $[20,21]$. The longest Ni nanorod was $1.35 \mathrm{Im}$, and the average length of $\mathrm{Ni}$ is $0.96 \mathrm{~lm}$ with $80 \%$ dis- tributing between 0.8 and $1.1 \mathrm{~lm}$ (Fig. 2c). The polycrystalline character of the elongated Ni nanorods is confirmed in Fig. 3, which shows a TEM image of one Ni/ CNF nanorod core/shell structure from the same sample as that in Fig. 2. The Ni nanorod fills the whole cavity of the CNF, with its length slightly shorter than that of the surround-ing CNF, due to some graphite layers located at the bottom of the CNF $[20,21]$. The contrast of the whole Ni nanorod is var- iable: the head (marked by " 1 ") is dark, but the tail (marked by " 2 ") is somewhat shaded, showing light gray regions. Fig. $3 b$ and c are the corresponding selected area electron diffraction patterns (EDPs) from areas " 1 " and " 2 ", respectively. The EDP from particle " 1 " is along [ $\left[\begin{array}{lll}1 & 1 & 0\end{array}\right]$ Ni showing a twin relation- ship, where a $\left\{\begin{array}{llll}1 & 1\end{array}\right\}$ twin boundary is parallel to the axis of the $\mathrm{CNF}$, as marked by a light blue line and an arrow in Fig. 3d. In contrast, the EDP from particle " 2 " is far away from [1 10 ] Ni and without twin diffraction pattern. The difference in EDPs from areas " 1 " and " 2 " indicates that they are differ- ent grains, which can further be verified by the dark-field TEM images in Fig. $3 \mathrm{~d}$ and e: the bright contrast in those dark-field images displays the contour of single grains, clearly showing the boundaries of grains " 1 " and " 2 " The polycrystalline character of the Ni nanorod is inher- ited from a former stage of the growth, as shown in Fig. 4. A typical Ni catalyst particle from a sample grown for $10 \mathrm{~min}$ was examined by a careful TEM analysis. Fig. 4a is a bright-field TEM micrograph showing a large $\mathrm{Ni}$ catalyst particle located on the graphite-like structure (corresponding to the bottom part of the CNF to be grown). The correspond- ing EDP is shown in Fig. 4b. The main part of the Ni particle was tilted to the [1 110$]$ zone axis showing dark contrast. However, there is a small part, with a light contrast (marked by an arrow), which indicates a different orientation. The dominant diffraction spots in Fig. $4 \mathrm{~b}$ indicate a twin relation- ship. Some extra diffraction spots marked by white arrow- heads (Fig. 4b) imply that the large Ni catalyst particle consists of additional grains besides the twin parts, the de- tails of which are further revealed by the dark-field images in Fig. $4 \mathrm{c}-\mathrm{f}$. The white contrast in those dark-field images clearly reveals that the large Ni particle at least consists of four parts, labeled " 1 " to " 4 " in Fig. 4c-f. Parts " 1 " and " 2 " have twin relationship with their twin boundary extending straight through the whole particle, from left to right, as marked by two white arrows. Besides the twin parts, two smaller grains, namely parts " 3 " and " 4 ", are also displayed. Their shapes, as shown by the white contrast, are quite irregular. The polycrystalline character of the nanoparticle is helpful for the growth of CNF because grain boundaries are known to be active sites for catalytic decomposition of carbon-containing precursors and a!so provide channels for enha nced carbon diffusion [22]. Different from the continuous 
feeding of gaseous-like catalytic precursors (nickelocene or ferrocene) through the catalyst pool at the base of CNTs [6], the elongated Ni nanorods filling the core of CNFs reported here originate only from solid Ni catalyst originally located in the tips of CNFs. The compressive pressure from graphene layers, which can be as large as rv40 GPa [9], probably extrudes the Ni catalyst particles, elongating them along the axes of CNFs. Subsequently, $\mathrm{Ni}$ nanorods are constrained in the core of CNFs by the compressive stress from adjacent graphene layers [17]. We note again that $\mathrm{Ni}$ nanorods are rarely sheathed in the core of CNFs with small diameters such as the thin CNF shown in Fig. 2 (right hand side) as well as in supporting information, SFig. 1a, and the short and thin CNFs in Fig. 5a. This is probably caused by the limited available Ni quantity in the small catalysts, which has also been con- firmed by growing CNFs from thinner Ni thin films of $10 \mathrm{~nm}$ or $20 \mathrm{~nm}[18,19]$, where there are no Ni nanorods found in the core of CNFs. With increasing the growth time to $85 \mathrm{~min}$, Ni nanorods appear to break down into several shorter individual ones, as seen in Figs. $1 \mathrm{f}$ and $5 \mathrm{a}$. The short Ni nanorods are encap- sulated in the hollow cavity of CNF, locating at intervals, from the bottom to the tip of the core of CNFs. The TEM image in Fig. 5a shows again that Ni nanorods are essentially encapsu- lated in CNFs with a diameter larger than rv0.20 Im, but seldom found in small CNFs with a diameter less than $0.20 \mathrm{Im}$, which is consistent with the observations in Fig. 2. Generally, the separated smaller Ni nanorods are single crystals, but two Ni nanorods connected together to form a relatively long Ni bicrystalline nanorod can also be found (Fig. $5 b$ ). Similar to previous observations of Fe-containing residuals in CNTs [23,24], a necking of particles, e.g., areas marked by white arrowheads in Fig. 5b and $\mathrm{c}$, is also found here. This strongly suggests that the division of the long nanorods is due to pref- erential breaking (as the CNF grows) in the necking areas where the nanorod diameter is smaller (lower mechanical strength). In general, the two diameters of the terminal edges of the encapsulated small Ni nanorods are smaller than that of their middle part inducing an oblong shape, which is caused by the necking effect prior and during the separation. The majority of CNFs have their lengths around 4-5 Im, with elongated Ni nanorods and empty spaces located in their cavities alternately (Fig. 5c). Instead of well-defined graphene caps between two adjacent metal particles encapsulated in CNTs [6], few random graphene layers are found in-between two $\mathrm{Ni}$ nanorods (see e.g., Fig. 5c), similar to those observed with the residual iron-containing catalysts confined in CNTs by CVD [23]. The crystallographic orientations of the encapsulated Ni nanorods in one CNF are confirmed by the corresponding EDPs (Fig. 6). Particle " 1 " and " 2 " have different orientations of [ $\left[\begin{array}{lll}1 & 1 & 0\end{array}\right]$ and $\left[\begin{array}{lll}1 & 0 & 0\end{array}\right]$, respectively, which again is similar to the observations concerning residual Fe catalyst particles in- side the core of CNTs [23]. However, particles " 2 " and " 3 " have the same [1 0 0] orientation, implying that they could be sep- arated from the same single crystal particle. The random ori- entation of the separated Ni nanorods may indicate that breaking primarily takes place at grain boundaries, as it is easier to separate grain boundaries than to break a single crystal, due to the weaker cohesion of the former [25]. The same orientation between the adjacent particles indicates that single crystal Ni nanorods could be further split or even rotated and twisted under the stresses from the CNFs during the growth process, which has previously been observed with iron-based catalysts [26]. It could explain why there are gen- erally more small nanorods in the core of CNFs than grains in the starting nanoparticle. 

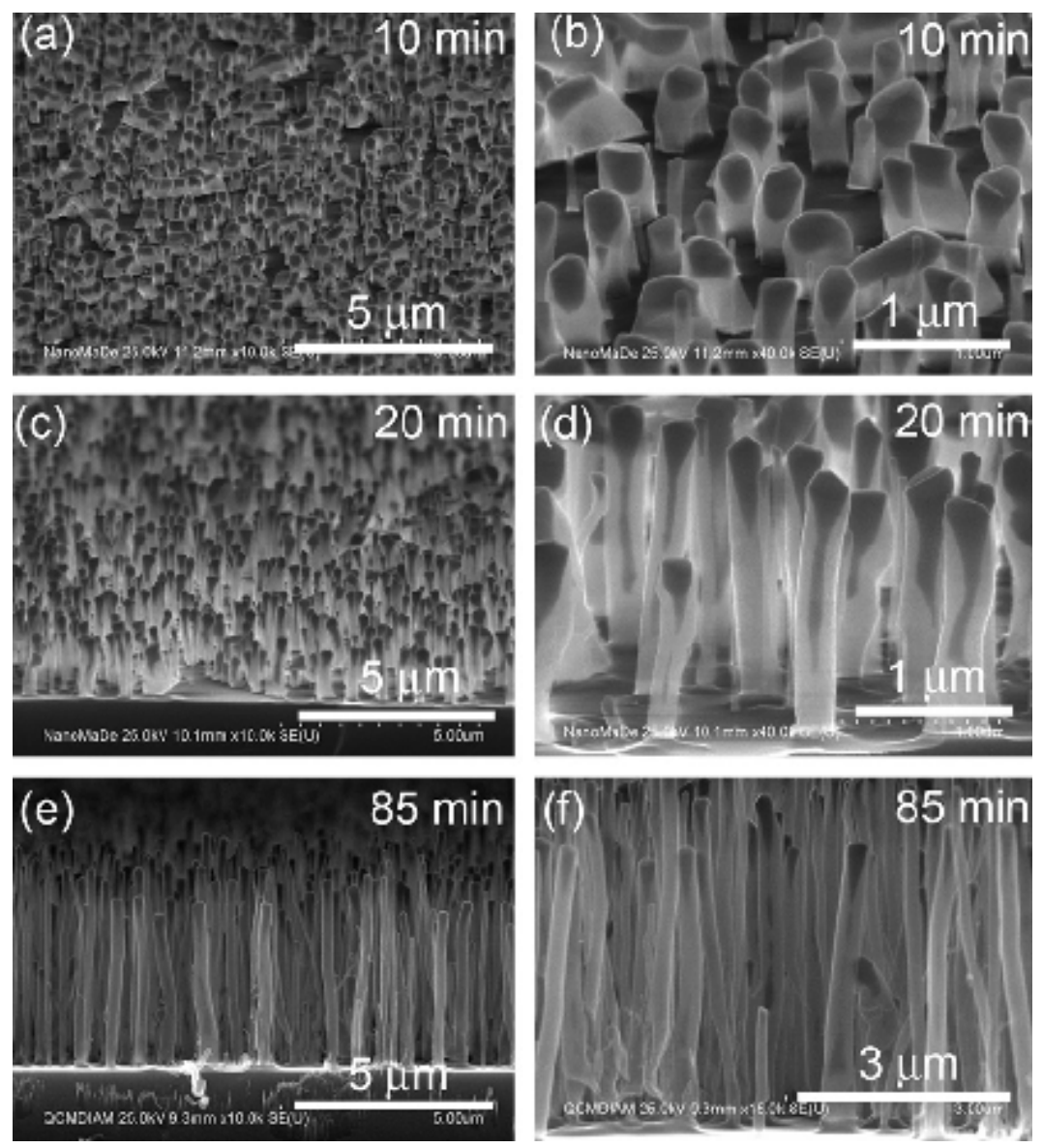

Fig. 1 - SEM images of CNFs grown for different times. Left-hand column: taken at low magnifications; right-hand column: magnified images. (a) and (b) Grown for 10 min. Ni catalysts show the characteristic inversed pear-like shape at the tips of CNFs. (c) and (d) Grown for 20 min. Ni catalysts change to a tadpole-like shape with the long tails confined in the core of CNFs to form a Ni/CNFs core/shell structure. (e) and (f) Grown for 85 min. Long Ni nanorods are broken into separated small individual

ones.
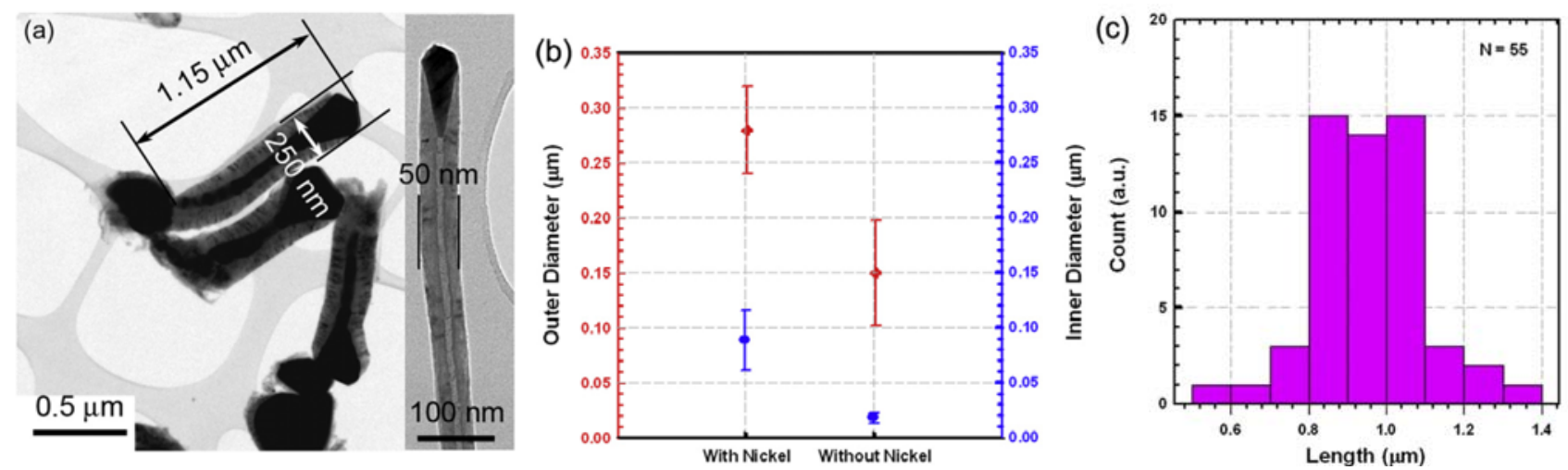

Fig. 2 - (a) Bright-field TEM image of CNFs grown for 20 min. Ni nanorods, showing a dark contrast, are elongated along the axis of the CNFs, and fill the whole cavity of the large CNFs with a diameter of $250 \mathrm{~nm}$. However, Ni nanorods do not fill small CNFs such as the one with a diameter of $50 \mathrm{~nm}$, as demonstrated by the inserted CNF picture in the right-hand part.(b) The outer and inner diameter of CNFs with and without Ni nanorods filied in the core of CNF.(c) Number of CNFs filled with Ni nanorods vs length of Ni nanorods after a 20 min growth time. 


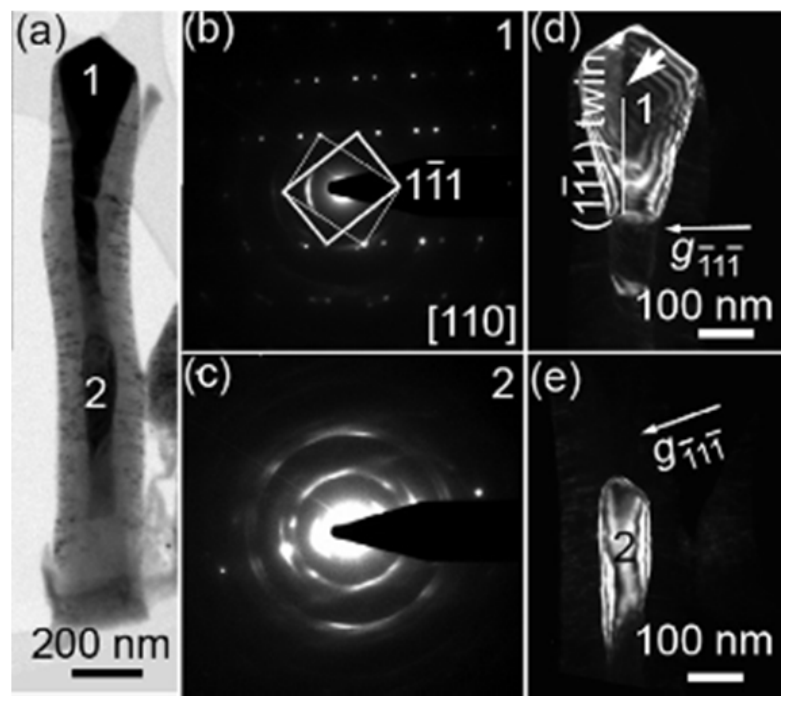

Fig. 3 - (a) A low magnification bright-field TEM image of one Ni/CNF core/shell structure grown for 20 min. A Ni nanorod is filling the whole core of the CNF, exhibiting a variable contrast along its length. The Ni nanorod is found to be slightly shorter than the CNF, due to the thick graphene layers occupying the base of the CNF. (b) and (c) Selected- area EDPs from the areas labeled " 1 " and " 2 " in (a), respectively. Different zone axes from the areas labeled " 1 " and " 2 " indicate that the long Ni nanorod is not a single crystallite, which can also be verified from the grain boundaries in the middle of the long Ni nanorod in the dark- field images in (d) and (e).

Fig. 7 is a schematic giving a summary of the growth process from the initial Ni particles to the final core/shell nanostructures. Two Ni particles with different sizes are shown, in order to compare the different nanostructures caused by the size effect of the starting Ni particles. After some short growth time by PECVD, e.g., 10 min, a thick graphite layer deposits at the base of the large Ni particles, with some graphite layers going up, surrounding the side surfaces of the large Ni particle (as verified by Fig. 4). The large Ni particle is raised up from the substrate by the graphite layers, but there is no spacing between the Ni par- ticle and graphite layers. However, in the same time, a CNF structure is already formed by a small Ni nanoparticle cata- lyst (the one on the right-hand side in Fig. $7 \mathrm{~b}$ ). The large Ni particle, is progressively extruded into the core of the CNF to form Ni/CNF core/shell (Fig. 7c and d), whereas, the small Ni particle stays at the tip of the CNF to form a normal CNF without Ni filling. In addition to the compressive stresses imposed on the side of the Ni nanorods by the CNF [9], there is also the upward tensile stress along the axis of CNFs, which results from the stress of the graphene layers pushing on the sides of the pear-like head of catalysts, as schematically illustrated by the green 1 and yellow arrows in Fig. 7d. Also, the electrostatic force F caused by the electric field during the PECVD growth process (black arrow on Fig. 7d) creates tensile stress along the axis of the CNFs, lifting the catalyst particles [17]. Consequently, the upward tensile stres- ses tend to raise the Ni nanorods, whereas the compressive radial stresses tend to retain the Ni nanorods, which finally results in the necking and fracture (Fig. 7e). This mechanism might also explain the presence of residual catalysts in many CNTs grown by CVD (omitting in the latter case the effect of the electric field). It is interesting to note that the growth orientation of CNFs adjusts itself parallel to the direction of the electric field (i.e., perpendicular to the substrate). In Fig. 6a, the initial stage of CNF growth, as marked by a red arrow, had been oblique to the substrate by rV77o, and was then corrected to the direction normal to the substrate by a rv13o rotation. The confined Ni nanorods changed to the same direction correspondingly, but they lag behind the direction change of the CNF. For example, the Ni par ticle labeled "4" on Fig. 6a has the same direction as the red arrow, although the $u$ pper part of the CNF su rrounding it has a!ready changed to the direction normal to the substrate. The reoriented adjustment of the CNF could be caused by the electrostatic force $\mathrm{F}$ along the axis of CNF, as discussed by Merkulov et al. [17]

\section{Conclusion}

Vertically aligned Ni/CNF core/shell nanostructures have been synthesized by PECVD. Ni nanorods filling the core of CNFs are extruded from large Ni catalyst particles during the tip-growth of CNFs, which appears totally different from the most encountered situation, where the metal confined inside the core of CNTs is continuously fed from a metal- containing gaseous precursor. In our case, the ratio of CNFs filled with Ni nanorods could reach rV70\%, in which $96 \%$ CNFs have an outer diameter larger than $0.2 \mathrm{~lm}$. More importantly, rv91\% of the Ni nanorods are continuous, pro- vided the growth time is adapted (typically 20 min in our growth conditions). Moreover, each Ni/CNF core/shell nano- structure is vertically-oriented, as well as isolated from the others, which is a different situation compared to previous results, where the vertical alignment was the consequence of a crowding effect, making CNTs/CNFs to be connected with each other by van der Waals forces in a bunch-like manner. Continuous Ni nanorods were broken into separate smal- ler ones with increasing growth time, forming another kind of core/shell structure. This phenomenon was explained by the combined effects/actions of the weaker grain boundaries, the twist or rotation of the Ni nanorods under the compres- sive stresses on their side walls, and the pulling stress along the axis of Ni nanorods. This result helps elucidating the common observation of residual catalyst particles in the core of CNTs synthesized by catalytic CVD, which was not clear so far. We believe that our results could be of interest in the field of nanodevices, particularly in magnetism and spintronics where such kind of organized core/shell structure could find applications. In addition, the same kind of growth process could be used to synthesize other core/shell nanostructures with different metals or metal carbides confined in the core of CNFs/CNTs.

\section{Acknowledgments}


This work was supported by the Region Ile-de-France in the framework of C'Nano IdF, France. Z.B.H. thanks Dr. G. Rizza and P.-E. Coulon at Laboratoire des Solides Irradie' s, Ecole Polytechnique, France for useful discussions. D.P. acknowl- edges support from the WCU (World Class University) pro- gram through the National Research Foundation of Korea funded by the Ministry of Education, Science and Technology (R31-2008-10029).
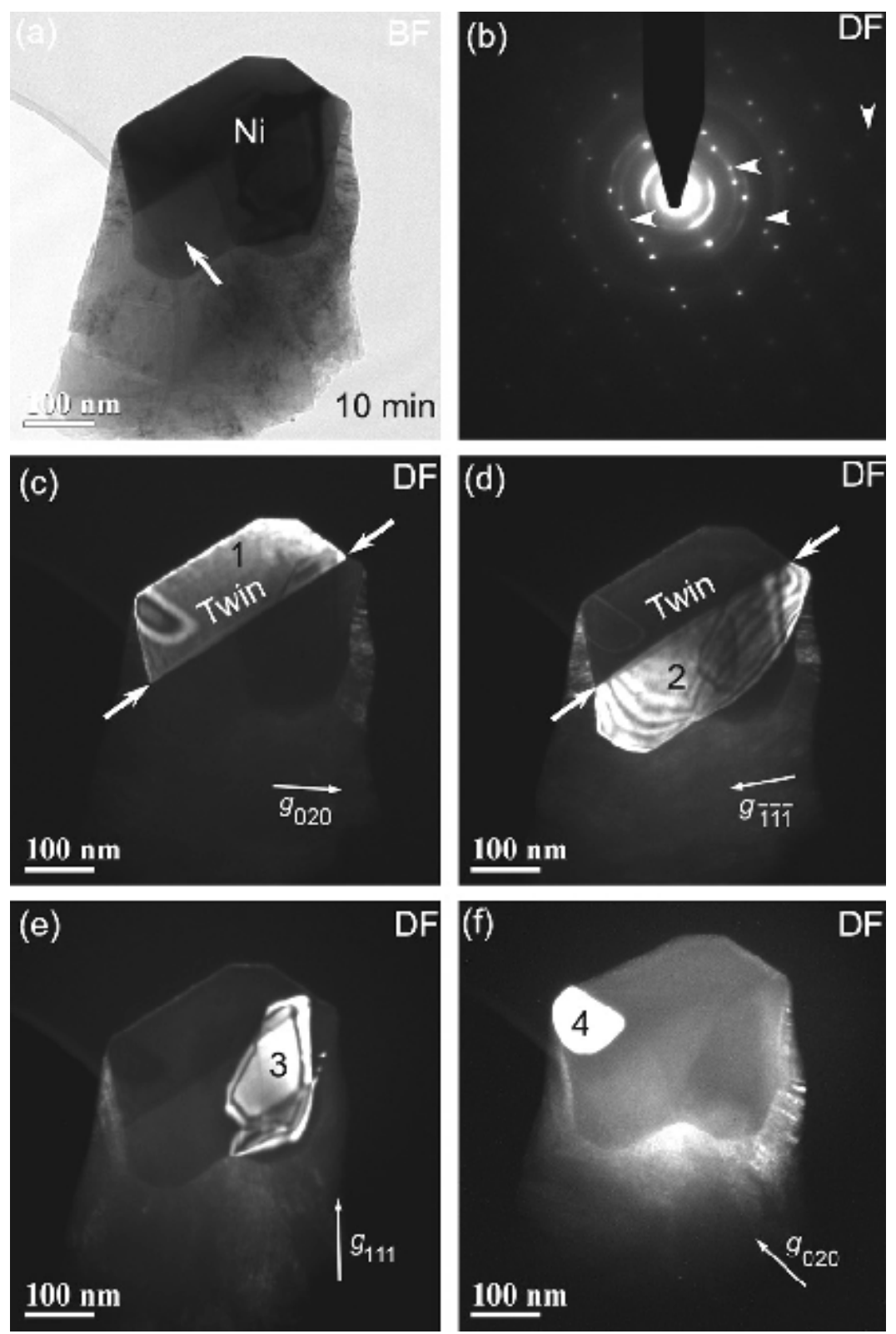

Fig. 4 - A typical Ni catalyst particle from a sample grown for 10 min. (a) Bright-field TEM image of the Ni catalyst particle on the graphite like structure. (b) The corresponding EDP reveals twin and polycrystalline characteristics. (c)-(f) Dark-field TEM images show four different crystallites in the large Ni particle, two of them being 

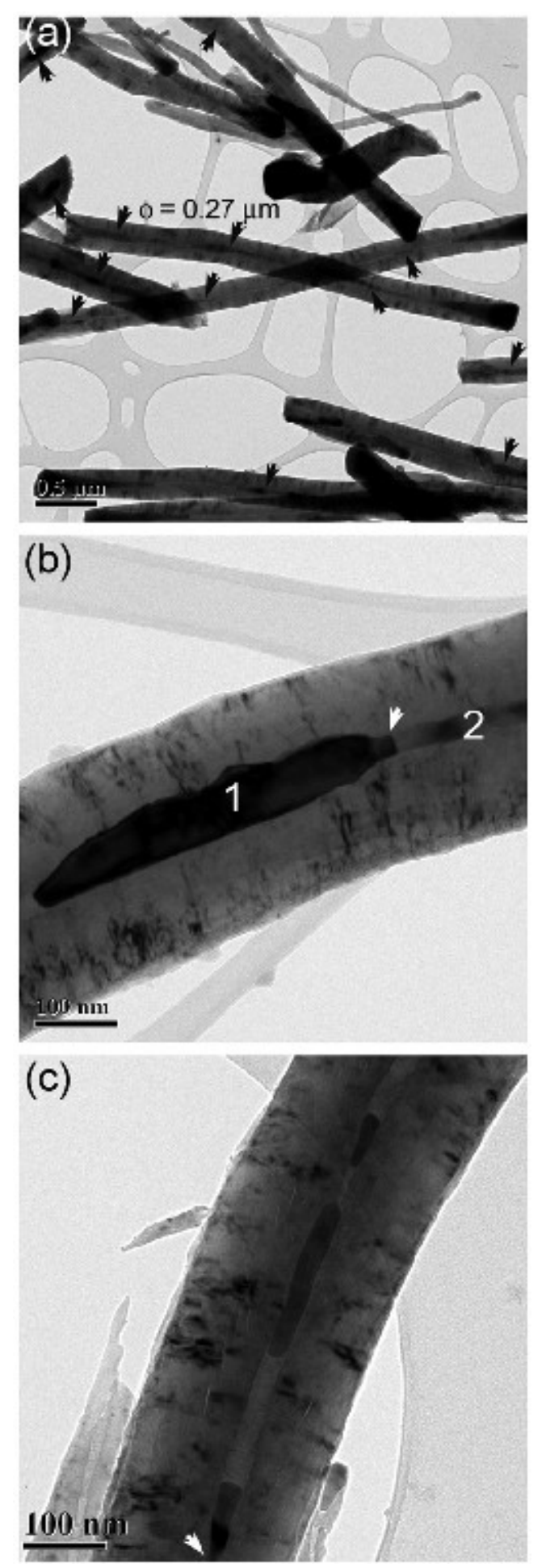

Fig. 5 - (a) A low magnification bright-field TEM micrograph of a sample grown for $85 \mathrm{~min}$. Note that thick CNFs with a diameter of $250 \mathrm{~nm}$ are now filled by separated Ni nanorods, as marked by black arrowheads. (b) An enlarged TEM bright field image shows one Ni nanorod confined in the core of a CNF. It consists of two crystallites, with crystallite "1" tilted to the zone axis to display it dark. The grain boundary between crystallites "1" and "2" is marked by a white arrowhead. (c) An enlarged TEM bright-field image shows a string of Ni nanorods separated by empty spaces without any thick graphene caps in-between the adjacent Ni nanorods. 

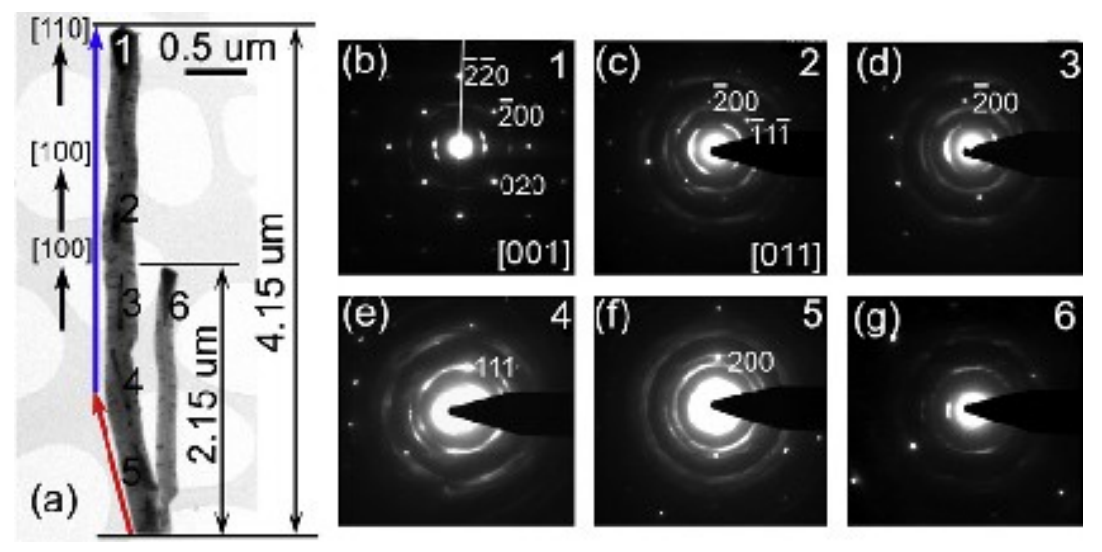

Fig. 6 - (a) Low magnification bright-field TEM micrograph including two CNFs sharing the same root (grown for 85 min). The crystal orientation of the encapsulated Ni nanorods " $1-3$ " is indicated on the left. (b)-(g) The corresponding EDPs from the nanoparticles. Catalysts on the tips of two CNFs, namely particles " 1 " and "6", have different orientation, as confirmed by comparing EDPs in (b) and (g) although they share the same growth root. Note that the growth direction of the longer CNF adjusts to the direction normal to the substrate after some time, as indicated by the red arrow followed by the blue one. (For interpretation of the references to color in this figure legend, the reader is referred to the web version of this article.)

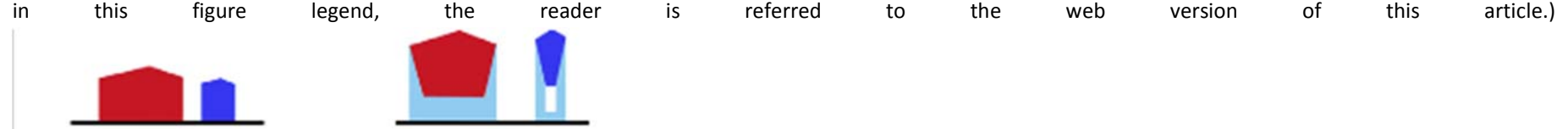

(a)

(b)

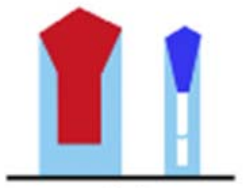

(c)

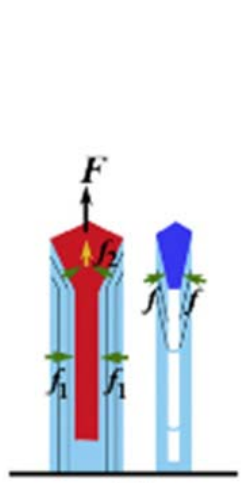

(d)

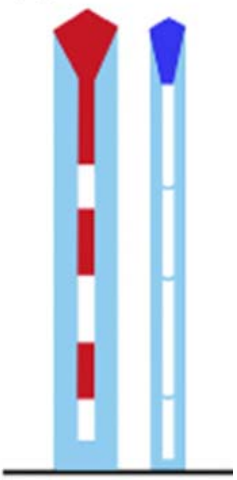

(e)

Fig. 7 - Schematic diagrams showing the process transforming Ni particles into nanostructures. Different Ni sizes produce different structure after growth. (a) Two Ni particles with different sizes. (b) Ni/CNF structures after a short growth time by PECVD. (c) and (d) Ni/CNF core/shell structure for the large Ni catalyst is formed due to the extrusion of the Ni tip by the CNF. (e) A long Ni nanorod is broken into separated individuals ones with increasing growth time due to the combined effects of stress and limited $\mathrm{Ni}$ quantity. The compressive lateral stress $\mathrm{f}_{1}$, the upward stress $\mathrm{f}_{2}$ (upward stress resulting from $\mathrm{f}_{1}$ in the cone-shaped tip part of the particle), and the electrostatic force $F$ caused by the electric field during PECVD growth are indicated in (d).

Supplementary data associated with this article can be found, in the online version, at doi:10.1016/j.carbon.2011.06.075.

\section{REFERENCES}

[1] Ajayan PM, lijima S. Capillarity induced filling in carbon nanotubes. Nature 1993;361:333-4.

[2] Han W, Fan S, Li Q, Hu Y. Synthesis of gallium nitride nanorods through a carbon nanotube-confined reaction. Science 1997;277:1287-9.

[3] Che R, Peng L, Duan X, Chen Q, Liang X. Microwave absorption enhancement and complex permittivity and permeability of Fe encapsulated within carbon nanotubes. Adv Mater 2004;16:401-5.

[4] Demoncy N, Ste' phan O, Brun N, Colliex C, Loiseau A, Pascard H. Filling carbon nanotubes with metals by the arc-discharge method: the key role of sulphur. Eur Phys J B 1998;4:147-57.

[5] Feng S, Zhao J, Du G, Song C, Song J, Zhu Z. Carbon nanotube- confined evolution of Co-Ni alloy nanowires with high- density lamellar twin boundaries. J Phys Chem 2008;C112:15247-52.

[6] Xiang R, Luo G, Qian W, Zhang Q, Wang Y, Wei F, et al. Encapsulation, compensation, and substitution of catalyst particles during continuous growth of carbon nanotubes. Adv Mater 2007;19:2360-3.

[7] Shpak AP, Kolesnik SP, Mogilny GS, Petrov YN, Sokhatsky VP, Trophimova LN, et al. Structure and magnetic properties of iron nanowires encased in multiwalled carbon nanotubes. Acta Mater 2007;55:1769-78.

[8] Kunadian I, Andrews R, Qian D, Mengu“ c, MP. Growth kinetics of MWCNTs synthesized by a continuous-feed CVD method. Carbon 2009;47:384-95. 
[9] Sun L, Banhart F, Krasheninnikov AV, Rodriguez-Manzo JA, Terrones M, Ajayan PM. Carbon nanotubes as high-pressure cylinders and nanoextruders. Science 2006;312:1199202.

[10] Hu J, Bando Y, Zhan J, Li C, Golberg D. Mg3N2-Ga: nanoscale semiconductor-liquid metal heterojunctions inside graphitic carbon nanotubes. Adv Mater 2007;19:1342-6.

[11] Sengupta J, Jana A, Singh NDP, Mitra C, Jacob C. Site-selective synthesis of in situ Ni-filled multi-walled carbon nanotubes using Ni(salen) as a catalyst source. Nanotechnology 2010;21:415605-1-6.

[12] Gao MR, Xu WH, Luo LB, Zhan YJ, Yu SH. Coaxial metal nano-/ microcables with isolating sheath: synthetic methodology and their application as interconnects. Adv Mater 2010;22:1977-81.

[13] Mu“ Iler C, Elefant D, Leonhardt A, Bu“ chner B. Incremental analysis of the magnetization behavior in iron-filled carbon nanotube arrays. J Appl Phys 2008;103:034302-1-5.

[14] Hayashi Y, Tokunaga T, Kaneko K, Henley SJ, Stolojan V, Carey JD, et al. Microstructure analyses of metal-filled carbon nanotubes synthesized by microwave plasmaenhanced chemical vapor deposition. IEEE Trans Nanotechnol 2006;5:485-90.

[15] Soldano C, Rossella F, Bellani V, Giudicatti S, Kar S. Cobalt nanocluster-filled carbon nanotube arrays: engineered photonic bandgap and optical reflectivity. ACS Nano 2010;4:6573-8.

[16] Meyyappan M, Delzeit L, Cassell A, Hash D. Carbon nanotube growth by PECVD: a review. Plasma Sources Sci Technol 2003;12:205-16.

[17] Merkulov VI, Melechko AV, Guillorn MA, Lowndes DH, Simpson ML. Alignment mechanism of carbon nanofibers produced by plasma-enhanced chemical-vapor deposition. Appl Phys Lett 2001;79:2970-2.

[18] He ZB, Maurice J-L, Lee CS, Gohier A, Pribat D, Cojocaru C-S. Nickel catalyst faceting in plasma-enhanced direct current chemical vapor deposition of carbon nanofibers. Ara J Sci Eng 2010;35:19-28.

[19] He ZB, Maurice J-L, Lee CS, Gohier A, Pribat D, Cojocaru CS. Etchant-induced shaping of nanoparticle catalysts during chemical vapour growth of carbon nanofibres. Carbon 2011;49:435-44.

[20] Cojocaru CS, Senger A, Normand FL. A nucleation and growth model of vertically-oriented carbon nanofibers or nanotubes by plasma-enhanced catalytic chemical vapor deposition. J Nanosci Nanotechnol 2006;6:1331-1-8.

[21] Cui H, Yang X, Simpson ML, Lowndes DH, Varela M. Initial growth of vertically aligned carbon nanofibers. Appl Phys Lett 2004;84:4077-9.

[22] Baraton L, He ZB, Lee CS, Maurice J-L, Cojocaru CS, Lee YH, Pribat D. On the mechanisms of precipitation of graphene on nickel their films. EPL, submitted for publication, Available from : arXiv:1106.3832.

[23] Wen CY, Huang CC, Cheng HZ, Lu HY. Orientation relations between carbon nanotubes grown by chemical vapour deposition and residual iron-containing catalysts. J Mater Sci 2008;43:123-31

[24] Behr MJ, Mkhoyan AK, Aydil ES. Orientation and morphological evolution of catalyst nanoparticles during carbon nanotube growth. ACS Nano 2010;4:5087-94.

[25] Sennour M, Lartigue-Korinek S, Champion Y, Hy“ tch MJ. Local strain analysis in twin boundaries in ultrafine grained copper. J Mater Sci 2008;43:3806-11.

[26] Behr MJ, Mkhoyan AK, Aydil ES. Catalyst rotation, twisting, and bending during multiwall carbon nanotube growth. Carbon 2010;48:3840-5. 